\title{
Editorial: Involvement of Autophagy in Determining the Developmental Fate of Ovarian Follicles
}

\author{
Jia-Qing Zhang ${ }^{1}$, Karin Lykke-Hartmann ${ }^{2,3,4}$ and Ming Shen ${ }^{5 *}$ \\ ${ }^{1}$ Institute of Animal Husbandry and Veterinary Science, Henan Academy of Agricultural Sciences, Zhengzhou, China, \\ ${ }^{2}$ Department of Biomedicine, C Aarhus University, Aarhus, Denmark, ${ }^{3}$ Department of Clinical Medicine, Aarhus University, \\ Aarhus, Denmark, ${ }^{4}$ Department of Clinical Genetics, Aarhus University Hospital, Aarhus, Denmark, ${ }^{5}$ College of Animal Science \\ and Technology, Nanjing Agricultural University, Nanjing, China
}

Keywords: follicular development, granulosa cell survival, oocyte aging, luteinization, hypoxia, autophagy, cell signalling

Editorial on the Research Topic

Involvement of Autophagy in Determining the Developmental Fate of Ovarian Follicles

Autophagy is an evolutionary conserved catabolic mechanism in eukaryotes that involves recycling of cytoplasmic proteins and organelles to maintain cellular homeostasis. Over the past decade, studies have demonstrated that autophagy governs an array of physiological processes in follicular development, ovulation and luteinization. This special issue contains a collection of six original research papers and three abstracts covering a broad range of topics providing novel insights into the mechanisms of autophagy in regulating the physiological functions of ovarian follicular cells.

Qiang Liu et al. explored the molecular relationships among FSH, progesterone production, lipid droplet (LD) degradation, and autophagy in porcine granulosa cells (GCs) (Liu et al.). They found that FSH activated the PI3K/AKT and SAPK/JNK pathways to stimulate BECLIN1 expression, leading to upregulated autophagy and LD degradation, which promoted progesterone production. Although FSH treatment could also significantly increase the mRNA levels of StAR and P450scc, inhibiting autophagy using chloroquine or JNK antagonist in FSH-treated GCs abolished FSHinduced progesterone production, while the expression of StAR and P450scc was not disturbed. This result further suggested that autophagy might play a role in providing for the increased substrate requirements resulting from $\mathrm{FSH}$-induced expression of steroidogenesis enzymes. Together, this study provides evidence demonstrating a novel role of autophagy in FSH-regulated steroid production.

Two articles by Tang et al. focused on the role of autophagy under hypoxic conditions in the rat ovary, firstly in the avascular granulosa layer of the developing follicle and secondly during the early formation of the corpus luteum (Tang et al.). The authors cultured the GCs from antral follicles and granulosa-lutein cells from developing corpora lutea under $3 \%$ oxygen or $\mathrm{CoCl}_{2}$ treatment to mimic hypoxia in vitro. The studies revealed that hypoxia induces in both in vitro culture systems HIF1alpha/BNIP3 signaling resulting in elevation of autophagy. Therefore, autophagy appears to be essential for GC survival during folliculogenesis and for the luteinisation of GCs after ovulation.

Follicular atresia is the ultimate fate of most ovarian follicles (>99\%) in mammalian ovary (Baker, 1963). Protein phosphorylation has been proven to be involved in regulating follicular atresia (Yang et al., 2020a). However, there have been very few studies aimed at characterizing the phosphoproteomic dynamics during follicular atresia. Utilizing TMT labeling, enrichment of phosphopeptides and LC-MS/MS analysis, Yang et al. conducted a proteomic/phosphoproteomic profiling of GCs from the healthy, slightly atretic, and atretic follicles in porcine ovaries. Several differentially expressed proteins (DEPs) and phosphorylated proteins (DEPPs) were identified as 
potential novel biomarkers to distinguish follicles of different atretic statuses. Based on mechanism research, they demonstrated that dephosphorylation of caspase six at Ser76 can increase the ratio of cleaved caspase6/caspase6 and lead to GCs apoptosis. Moreover, the phosphoproteomic data revealed some kinase regulatory networks of autophagic signaling. Compared with atretic follicles, the activation of PIK3C3 in healthy follicles was predicted to inhibit autophagy by catalyzing phosphorylation of autophagic regulators such as SQSTM1 (at S272) and AMBRA1 (at S52), indicating that autophagy might be involved in the atretic process. These findings might provide novel explanations regarding the regulation of follicular atresia.

It is still a matter of debate as to whether autophagy, not only facilitates corpus luteum formation by preventing apoptosis, but also promotes luteolysis through excessive cell destruction. The switch mechanism of "autophagic survival" and "autophagic death" during luteinization and luteolysis remains to be elucidated. Przygrodzka et al. explored the autophagic signaling pathways required for luteinization and luteolysis in steroidogenic cells of the corpus luteum. Their results indicated that luteotrophic signaling via a LH/PKA/MTOR pathway inhibits, while luteolytic signaling via a PGF2 $\alpha / \mathrm{Ca} 2+/ \mathrm{AMPK}$ pathway activates key signaling pathways involved in luteal cell autophagosome formation. They demonstrated that autophagy-related signaling is upregulated during luteolysis, accompanied by cellular death. These findings extend the current knowledge on two prominent metabolic pathways, AMPK and PKA/MTOR signaling, in the maintenance or regression of the corpus luteum.

The autophagic activity has been found to decrease with age, likely leading to the accumulation of damaged macromolecules and organelles. Peters et al. investigated whether autophagy is involved in age-related oocyte quality decline, which is considered as a major contributor to female infertility. Characteristics of autophagy dysfunction, including decreased autophagosomes, decreased lysosomes, and amphisome accumulation, were detected in the aged oocytes. Interestingly, there are no significant differences in the expression of macroautophagy related proteins between young and aged mouse oocytes. However, a growing number of large autophagic puncta $\left(>10 \mu \mathrm{m}^{2}\right)$ was observed in aged oocytes. Comprehensive image-based analysis revealed colocalization of

\section{REFERENCES}

Baker, T. G. (1963). A Quantitative and Cytological Study of Germ Cells in Human Ovaries. Proc. R. Soc. Lond. B Biol. Sci. 158, 417-433. doi:10.1098/ rspb.1963.0055

Yang, F., Chen, Y., Liu, Q., Dai, S., and Zeng, S. (2020a). Dynamics and Regulations of BimEL Ser65 and Thr112 Phosphorylation in Porcine Granulosa Cells during Follicular Atresia. Cells 9, 402. doi:10.3390/cells9020402

Conflict of Interest: The authors declare that the research was conducted in the absence of any commercial or financial relationships that could be construed as a potential conflict of interest. biomarkers of ensosomes and autophagosomes, representing the formation of amphisomes. In addition, aged oocytes exhibit decreased numbers of lysomes, while lysosomal inhibition promotes the formation of large amphisomes. These findings provide a novel model linking the compromise of lysosomal function and the accumulation of amphisomes to the mechanism of oocyte aging.

Taken together, these research articles introduce readers to novel insights and important perspectives on the relationship between autophagy and ovarian physiology. We hope that this Research Topic will serve as a platform to initiate new research questions and innovative ideas in the field of female reproduction.

\section{AUTHOR CONTRIBUTIONS}

MS was the Guest editor of this Research Topic, inviting coeditors J-QZ and KL-H working with them to define the subjects to be treated. They identified and invited leaders in specific research fields to contribute their work to the Research Topic. They acted as handling editors of manuscripts in the topic. MS wrote the Editorial with input from the other co-editors.

\section{FUNDING}

This work was supported by The National Natural Science Foundation of China (No. 31972564; No. 31972571; No. 31630072; No.31601939), a project funded by the Priority Academic Program Development of Jiangsu Higher Education Institutions (No. DKQB201903), Fund for Distinguished Young Scholars from Henan Academy of Agricultural Sciences. (No. 2021JQ07), Postgraduate Research and Practice Innovation Program of Jiangsu Province (No. KYCX20_0602), the National Major Project for Breeding of Transgenic Pigs (2016ZX08006001-003).

\section{ACKNOWLEDGMENTS}

The authors would like to thank all the authors who contributed to this Research Topic as well as the reviewers of the manuscripts for their efforts.

Publisher's Note: All claims expressed in this article are solely those of the authors and do not necessarily represent those of their affiliated organizations, or those of the publisher, the editors and the reviewers. Any product that may be evaluated in this article, or claim that may be made by its manufacturer, is not guaranteed or endorsed by the publisher.

Copyright (c) 2021 Zhang, Lykke-Hartmann and Shen. This is an open-access article distributed under the terms of the Creative Commons Attribution License (CC BY). The use, distribution or reproduction in other forums is permitted, provided the original author(s) and the copyright owner(s) are credited and that the original publication in this journal is cited, in accordance with accepted academic practice. No use, distribution or reproduction is permitted which does not comply with these terms. 